\title{
Efectos de una unidad didáctica de mimo basada en el modelo de Educación Deportiva sobre la interculturalidad
}

\author{
Effects of a unit of mime based on the Sports \\ Education model on interculturality
}

\author{
Antonio Méndez-Giménez ${ }^{1 *}$, Federico Puente-Maxera ${ }^{1}$ y Diego Martínez de Ojeda Pérez ${ }^{2}$ \\ 1 Departamento de Ciencias de la Educación. Universidad de Oviedo \\ 2 CEIP Profesor Enrique Tierno (Lobosillo, Murcia)
}

Resumen: El propósito de este estudio fue conocer el impacto generado por una unidad didáctica de mimo basada en el Modelo de Educación Deportiva (MED) sobre la competencia intercultural del alumnado de primaria. Participaron 28 estudiantes $(M=10.75 ; D T=.75)$ de quinto curso de educación primaria pertenecientes a un colegio ubicado en la Región de Murcia (España). Se desarrolló un diseńo cuasiexperimental con medidas pretest y postest, de naturaleza cuantitativa y cualitativa. La información fue recabada mediante cuestionarios y entrevistas al alumnado, así como diarios y entrevistas a los docentes. Los resultados mostraron mejoras en la respuesta emocional positiva, así como en la ayuda, y las relaciones entre iguales de distintas culturas y países. Se discute también la incidencia de los elementos metodológicos, la comunicación y el clima social del aula sobre el desarrollo de la competencia intercultural. Se sugieren líneas de investigación futura al objeto de perfeccionar el modelo para el desarrollo de la competencia intercultural.

Palabras clave: Competencia intercultural, educación física, modelos de enseñanza, educación deportiva.
Abstract: The aim of this study was to assess the impact of a Sport Education unit on elementary school students' intercultural competence. The program involved the participation of 28 fifth-grade students $(M=10.75 ; D T$ $=.75$ ) from a state school in Region of Murcia, Spain. A quasi-experimental design, pretest and posttest (of quantitative and qualitative nature) measures, was used. The data included students' questionnaires and interviews, and teachers' interviews and diary notes. Results indicated that students improved on positive emotional responses, as well as providing help and having better relations with their peers from different cultures/countries. The incidence of methodological elements, communication and the social climate of the classroom on the development of intercultural competence is also discussed. Lines of future research are suggested in order to improve the model for the development of intercultural competence.

Key words: Intercultural competence, physical education, instruction models, sport education.

\section{Introducción}

Una educación inclusiva es aquella que atiende a la diversidad de necesidades de todos los estudiantes, generando mayor participación en el aprendizaje y reduciendo el riesgo de exclusión (UNESCO, 2005). En ese amplio espectro de necesidades, se encuentra la inclusión de estudiantes con antecedentes culturales distintos al del grupo dominante; esto es, una educación verdadera y de calidad debe contemplar el hecho intercultural. Desde la pedagogía intercultural, se ha planteado como objetivo que el alumnado comprenda y acepte la diversidad existente, disipando ideas de otredad tan presentes y extendidas en la sociedad actual. La educación intercultural, como concepto inacabado y en permanente cambio, busca promover la igualdad de oportunidades en todo el alumnado a través de experiencias educativas que le ayude a ser culto y respetuoso con los demás (Banks, 2002). Implica, asimismo, modificaciones en aproximaciones, contenidos y estrategias educativas, de modo que el aprendizaje, como variable que define la educación inclusiva (Rodríguez,

Dirección para correspondencia [Correspodence address]: Antonio Méndez-Giménez. E-mail: mendezantonio@uniovi.es
2015), debe incardinar nuevas estrategias metodológicas que den respuesta a las necesidades del alumnado. Esto supone asumir el desarrollo de un nuevo complejo competencial en el que se incluyan capacidades con valor añadido (Santos y Lorenzo, 2015), entre las que destacan la interacción, la comunicación y la sensibilidad interculturales. Considerando esta última, atendemos a la conceptualización de Chen y Starosta (1996) quienes sitúan a la sensibilidad intercultural dentro de la dimensión afectiva de la competencia comunicativa intercultural, y definen aquella como la "habilidad para desarrollar emociones hacia la comprensión y valoración de las diferencias culturales orientadas a promover comportamientos apropiados y eficaces en la comunicación intercultural" (Chen y Starosta, 2000).

Haciendo una revisión sobre las investigaciones realizadas desde una mirada intercultural, hallamos numerosos estudios que han considerado el desarrollo de la competencia intercultural (Hernández, 2011; Malik, 2002; Pérez, 2013) o aspectos de la misma, como es el caso de la mencionada comunicación (Padró, 2004; Rodrigo, 1996; Vilà, 2003; 2005) y sensibilidad interculturales (Arslan, Günçavdi y 
Polat, 2014; Chongruska, Prinyapola, Wadengb y Padungponga, 2010; Sanhueza y Cardona, 2009). Dichos estudios han ido dirigidos a distintas etapas educativas, ya sea primaria (Pérez, 2013; Sanhueza y Cardona, 2009), secundaria (Muniategi, Sianesb y López, 2014) o terciaria (Arslan et al.,2014), así como a distintas áreas del conocimiento (sociolingüística, filosófica, rítmico-musical, etc.). Dentro de las propuestas existentes, destacan las que abogan más por la medición (con una clara intención diagnóstica) u otras dirigidas a colectivos particulares, de matiz compensatorio o folclórico y con muy escasa voluntad de enmienda. Propuestas, en definitiva, que ahondan muy poco en variables metodológicas o estilos de enseńanza-aprendizaje, ya que, en el mejor de los casos, permiten extraer conclusiones vagas sobre aprendizaje cooperativo lo que, a día de hoy, es una obviedad constatada. Los resultados más destacados apuntan a mejoras en las relaciones sociales (Derri,Kellis, Vernadakis, Albanidis, y Kioumourtzoglou, 2014), el conocimiento intercultural (Hernández, 2011) y las actitudes (Chongruskaet al.,2010).

No obstante, y más allá de la abultada mayoría de autores que confirman los beneficios derivados de las prácticas interculturales, son escasas las experiencias existentes desde el área de la Educación Física (EF) (Kouli y Papaioannou, 2009). En dicho campo, numerosas investigaciones han evidenciado la eficacia de determinados modelos pedagógicos sobre el clima y las interacciones sociales. Modelos que van desde el Aprendizaje Cooperativo (Barrett, 2000; 2005; Dyson, 2002; Fernández-Río, 2003; Velázquez, 2010) al Modelo Comprensivo (Dyson, 2005; Mandigo y Corlett, 2010; Sheppard, 2014; Thorpe, 1992), pasando por el Modelo de Responsabilidad Social e Individual (Gordon, 2009; Hellison, 2011). En ese proceso de cristalización metodológica destaca también el Modelo de Educación Deportiva (MED), cuyos valedores han cotejado su potencial a la hora de encauzar vías de interacción social (Brock, Rovegno y Oliver, 2009; Cruz, 2008; García-López, Gutiérrez, González-Víllora y Valero, 2012; MacPhail, Kirk y Kinchin, 2004; O’Donovan, 2003). Los aspectos más característicos del MED son (Siedentop, Hastie y Van Der Mars, 2011): (1) las temporadas, que suponen la estructura temporal de la unidad didáctica; (2) la afiliación, el alumnado se agrupa a través de equipos; (3) competición formal; (4) evento final, que supone el desenlace de la unidad; (5) registro de datos; y (6) festividad, ya que la unidad se desarrolla a través de un ambiente lúdico y divertido.

Por su parte, pocas han sido las intervenciones de carácter experimental que se hayan centrado en explorar el impacto de la expresión corporal sobre el rendimiento del alumnado en Educación Física. Entre las existentes, figuran propuestas basadas en la danza (Calderón, Hastie, Liarte y Martínez de Ojeda, 2013; Richardson y Oslin, 2003) o el mimo (Méndez-Giménez, Martínez de Ojeda, y Valverde, 2017a; 2017b). Los resultados más destacados certificaron el potencial de la expresión corporal para obtener mejoras en las habilidades sociales, la inteligencia emocional y las relaciones interpersonales. Todavía, son escasos los estudios que hayan examinado la incidencia de este tipo de programas en contextos con tasas elevadas de inmigrantes, considerando la percepción del alumnado en el desarrollo de la sensibilidad y el comportamiento intercultural. Tal es así que, atendiendo a las variables referidas en este trabajo, solo encontramos un estudio comparativo (Derri et al., 2014) dirigido a medir el comportamiento intercultural del alumnado de primaria en la EF. El comportamiento en cuestión aludió al desarrollo social y moral de los estudiantes (en este caso, griegos y extranjeros) en contextos multiculturales (Kellis Vernadakis, Albanidis, Derri, y Kourtesses, 2010). Como resultado, los estudiantes que participaron en el programa intercultural (PIEF) obtuvieron mejoras en las relaciones y en la interacción respecto a quienes lo hicieron bajo las premisas del programa tradicional. El PIEF fue diseñado desde el enfoque del aprendizaje cooperativo, destacando la valía de uno de sus elementos más característicos, a saber, la formación de grupos heterogéneos. Por su parte, el tratamiento de la sensibilidad intercultural en el área de la EF es muy limitado. Encontramos el estudio realizado por Burden, Hodge, O’Bryant, y Harrison (2004) dirigido a la formación del profesorado en EF, en el que se prescinde de la tan necesaria perspectiva del alumnado.

En cualquier caso, los diseños de intervención en los casos referidos no han sido modelados con el rigor metodológico que exigen variables de esta índole. Resulta difícil encontrar propuestas que se posicionen desde el prisma de algunos de los modelos pedagógicos citados anteriormente. Quizá la experiencia transnacional realizada por Hastie, Farias y Gutiérrez (2013) se aproxime más a nuestro cometido, en cuanto parten de las premisas del MED. Aun así, se aleja de nuestras pretensiones habida cuenta de las decisiones tomadas en la medición y obtención de información. A efectos de superar estas limitaciones, en esta investigación hemos tratado de analizar en qué medida un plan de intervención basado en los principios del MED, y en los que se introduce el mimo como contenido central, puede mejorar la competencia intercultural del alumnado en relación a la sensibilidad y comportamiento interculturales. Analizaremos cómo se desarrollan las relaciones sociales, conociendo la percepción del alumnado y profesorado sobre los encuentros interculturales, e identificando aquellos elementos didácticos más relevantes en clave intercultural. 


\section{Método}

\section{Enfoque}

La investigación se adscribe a un enfoque metodológico mixto, tanto cualitativo como cuantitativo que pretende dar voz tanto al alumnado como al profesorado inmerso en un proceso de enseñanza aprendizaje. Parte de un diseño cuasiexperimental descriptivo en el que fueron comparadas las medidaspretest - postest del alumnado y profesorado de tercer ciclo de educación primaria en su correspondiente centro educativo.

\section{Participantes}

Participaron en el estudio 28 alumnos y alumnas de quinto curso de educación primaria de un colegio público de la Región de Murcia, ubicado en la localidad de Lobosillo. El grupo fue seleccionado por su disponibilidad y alto grado de diversidad étnica y cultural (mayormente, alumnado de origen marroquí) alcanzando un porcentaje del $54 \%$. Sus edades oscilaban entre los 10 y 12 años $(M=10.75 ; D T=.75)$. En cuanto al género, la distribución se hallaba equilibrada, habiendo un total de 15 chicas y 13 chicos. Dos maestros especialistas en Educación Física impartieron docencia. Uno con experiencia respecto al uso del MED (lleva trabajando con esta metodología desde hace más de 4 años) y otro inexperto. El estudio contó con el consentimiento tanto del centro educativo como de los padres y madres del alumnado participante.

\section{Diseño}

Se implementó una unidad didáctica de mimo de 10 sesiones bajo las premisas del MED. Los 28 participantes fueron distribuidos en cinco grupos (heterogéneos en cuanto a género y diversidad étnico-cultural). Atendiendo a las premisas del modelo, cada alumno asumió una responsabilidad dentro de su grupo (director, presentador, guionista 1, guionista 2, y preparador físico), así como el rol propio de la obra a representar, es decir, el personaje. Igualmente, los equipos debieron realizar la labor de jueces (duty team) para valorar las obras representadas, tanto ajenas como propias, a partir de una hoja de registro previamente enseñada. La secuencia didáctica atravesó las siguientes fases: (a)introductoria, donde se dio a conocer el contenido, se organizaron los grupos, etc.; (b) dirigida, en la que el docente empleó métodos más directivos para impartir los contenidos, (c) de práctica autónoma, en la que los equipos (compañias) debían tomar sus propias decisiones y resolver problemas; (d) de competición formal, en la que se sucedieron una serie de representaciones objeto de valoración; y (e) el evento final, en el que los alumnos representaron su obra ante el alumnado de Educación Infantil, quien acudió en calidad de espectador.
Asimismo, al finalizar las actuaciones, los estudiantes de quinto impartieron un pequeño taller por grupos a los estudiantes de educación infantil en el que enseñaron las rutinas del mimo.

\section{Instrumentos}

Percepción de los estudiantes

Sensibilidad Intercultural. Se utilizó la Escala de Sensibilidad Intercultural (Chen y Starosta, 2000), adaptada por Sanhueza (2010), compuesta por 10 ítems valorados a partir de una escala tipo Likert de cinco puntos ( $1=$ Nunca es cierto, $2=$ Casi nunca es cierto, $3=A$ veces es cierto, $4=B$ astantes veces es cierto, $5=$ Siempre es cierto). El alfa de Cronbach obtenido por la autora fue de $\alpha=.75$. En cada ítem los estudiantes debían asignar dos valores, dando su opinión respecto a dos momentos: pretest (aquello que pensaban antes de trabajar la unidad) y postest (su opinión una vez concluida la intervención). Se establecieron dos factores: (1) respuestas emocionales positivas y (2) respuestas emocionales negativas. Una puntuación alta indicaría que el sujeto posee una muy buena sensibilidad intercultural y, por lo tanto, manifiesta respuestas emocionales positivas hacia otros culturalmente distintos.

Comportamiento Intercultural. Por otro lado, se administróla Escala de Autoevaluación del Comportamiento del Estudiante (Kellis et al., 2010), validada para evaluar el desarrollo moral y social de los estudiantes en contextos multiculturales y orientada a medir el comportamiento intercultural de los estudiantes. Su versión original consta de 18 ítems relacionados con cinco habilidades sociales: irresponsabilidad; recompensa; ayuda; familiaridad/relaciones; y metas. Se realizó una traducción-retrotraducción de los ítems de la versión de esta herramienta siguiendo las recomendaciones de Hambleton, Merenda, y Spielberger (2005). Se realizaron adaptaciones que afectaron a la terminología (mayor claridad y concisión) y a la organización de los ítems. El alfa de Cronbach de cada una de las subescalas en el estudio de Kellis et al. (2010) fue, respectivamente, $\alpha=.78$ (metas); $\alpha=.88$ (relaciones); $\alpha=.76$ (recompensa); $\alpha=.71$ (ayuda); y .73 (irresponsabilidad). Asimismo, se descartó la dimensión "metas" al ser considerada irrelevante, resultando un total de 14 ítems. El instrumento utilizó una escala tipo Likert de 1 "nunca" a 5 "siempre".

Entrevistas. Una vez concluida la intervención, se realizaron entrevistas en grupos de cinco-seis estudiantes tal y como recomiendan Ennis y Chen (2012), partiendo de los equipos previamente establecidos para el desarrollo de la unidad. De este modo, se registraron un total de cinco entrevistas semiestructuradas, dirigidas por los docentes correspondientes, en las que los respondientes aportaban su conocimiento acerca del programa desarrollado (Bisquerra, 2004). Los guiones de las entrevistas fueron revisados por cuatro expertos (dos doctores y dos maestros con, al menos, 10 años de experiencia). 
Percepción de los docentes

Entrevistas. Se realizaron entrevistas semi-estructuradas (Cohen y Manion, 2002) de manera individual, a cada uno de los profesores participantes y en tres momentos: antes, durante y después de la intervención. Las entrevistas, cuyos guiones fueron revisados por dos expertos (doctores), incidieron en cuestiones relacionadas con la interculturalidad, el clima social, los aspectos metodológicos y otras incidencias de consideración. Las entrevistas fueron registradas en archivos de audio y posteriormente transcritas.

Diarios. Los docentes involucrados en la intervención registraron las observaciones, impresiones e incidencias más relevantes encontradas en cada sesión. Dichos comentarios respondieron a criterios previamente establecidos en la investigación (Erickson, 1989). El diario se introdujo como una guía para la reflexión práctica favoreciendo conexiones teórico-prácticas de relevancia (Porlán y Martín, 1991).

\section{Análisis de datos cuantitativos}

Los datos fueron analizados de forma diferente, considerando el carácter cuantitativo y cualitativo de la información. Los datos cuantitativos fueron analizados mediante el paquete estadístico SPSS (versión 22.0), y las técnicas utilizadas fueron descriptivos y frecuencias, así como la inferencia estadística (comparación de rangos). Se analizaron las medias del grupo respecto a la fase pretest, en primer lugar, y postest, en segundo, con objeto de contrastar los efectos de la intervención. La Tabla 1 recoge la fiabilidad de los dos cuestionarios administrados al alumnado, así como de cada uno de sus factores, tanto en momentos pretest como postest. Considerando la baja fiabilidad (Alfa de Cronbach < .70) obtenida en la Sensibilidad Intercultural, su factor Respuesta Emocional Negativa, así como en la Relación y la Recompensa del Comportamiento Intercultural, tanto en momentos pretest como postest, no se realizó ningún análisis posterior con esos datos.

Tabla 1. Alfas de Cronbach de los cuestionarios empleados en Pretest y Postest.

\begin{tabular}{lcc}
\hline & Pretest & Postest \\
\hline Sensibilidad Intercultural & .61 & .61 \\
REP & .79 & .71 \\
REN & .51 & .52 \\
Comportamiento Intercultural & .79 & .65 \\
Ayuda & .77 & .61 \\
Irresponsabilidad & .64 & .69 \\
Relación & .67 & .34 \\
Recompensa & .32 & .51 \\
\hline
\end{tabular}

Se solicitó la prueba de Kolmogorov-Smirnov para valorar la normalidad de las variables, obteniéndose niveles de Sig.< .05. Por tanto, en los análisis subsiguientes de los cuestionarios se emplearon pruebas no paramétricas. Para el análisis de las puntuaciones pretest y postest se empleó la prueba de Rangos de Wilcoxon para 2 muestras relacionadas. Para el estudio y comparación entre géneros de las valoraciones recogidas en las diferentes variables se empleó la prueba $U$ de Mann Whitney de muestras independientes.

\section{Análisis de datos cualitativos}

Los datos cualitativos, más cuantiosos, fueron analizados de forma manual, haciendo uso de técnicas inductivas, a partir de la síntesis, codificación y comparación de las respuestas de los participantes (tanto profesorado como alumnado) mediante procesos de reducción y exposición de la información y, finalmente, extracción de conclusiones (Massot, Dorio y Sabariego, 2004). Tras varias lecturas, la información fue segmentada en una serie de ideas o percepciones, las cuales fueron codificadas y clasificadas en una serie de categorías y sub-categorías emergentes. En un primer momento, las categorías extraídas fueron cuatro: (1) Actitudes del alumnado, (2) Elementos metodológicos y su influencia en la interculturalidad, (3) Efecto de la comunicación sobre la interculturalidad y el liderazgo, y (4) Clima social de aula e interculturalidad. Finalmente, se decidió suprimir la categoría "Actitudes del alumnado" al no presentar información suficiente, directa y relevante sobre la variable de estudio. Las tres categorías resultantes fueron seleccionadas para el estudio.

\section{Resultados}

\section{Resultados cuantitativos}

La Tabla 2 presenta los estadísticos descriptivos de la subescala Respuesta emocional positiva (REP) de la Sensibilidad Intercultural así como del Comportamiento Intercultural y de sus Factores analizados en cada una de las administraciones de los cuestionarios y en función del género.

Tabla 2. Estadísticos descriptivos dela dimensión REP (Sensibilidad Intercultural) y Comportamiento Intercultural y sus Factores (Ayuda e Irresponsabilidad) de la muestra total, varones y mujeres.

\begin{tabular}{lcccc}
\hline & \multicolumn{2}{c}{ Pretest } & \multicolumn{2}{c}{ Postest } \\
\cline { 2 - 5 } & $M$ & $D T$ & $M$ & $D T$ \\
\hline Respuesta Emocional Positiva & & & & \\
\hline N total & 4.07 & .80 & 4.56 & .49 \\
Varones & 3.71 & .84 & 4.29 & .61 \\
Mujeres & 4.37 & .66 & 4.77 & .22 \\
\hline
\end{tabular}




\begin{tabular}{lcccc}
\hline & \multicolumn{2}{c}{ Pretest } & \multicolumn{2}{c}{ Postest } \\
\cline { 2 - 5 } & $M$ & $D T$ & $M$ & $D T$ \\
\hline Comportamiento Intercultural & & & & \\
\hline N total & 3.54 & .62 & 3.87 & .44 \\
Varones & 3.38 & .77 & 3.85 & .59 \\
Mujeres & 3.68 & .42 & 3.89 & .29 \\
\hline Ayuda & & & & \\
\hline N total & 3.87 & 1.05 & 4.31 & .75 \\
Varones & 3.62 & 1.13 & 4.16 & .90 \\
Mujeres & 4.08 & .98 & 4.43 & .61 \\
\hline Irresponsabilidad & & & & \\
\hline N total & 1.51 & .78 & 1.61 & .99 \\
Varones & 1.74 & .95 & 1.79 & 1.20 \\
Mujeres & 1.28 & .50 & 1.45 & .74 \\
\hline
\end{tabular}

\begin{tabular}{lc}
\hline & Postest - Pretest \\
\hline Mujer Z & -2.14 \\
Sig. asintót. (bilateral) & .03 \\
\hline Ayuda & \\
\hline N total Z & -2.86 \\
Sig. asintót. (bilateral) & .00 \\
Varón Z & -1.90 \\
Sig. asintót. (bilateral) & .05 \\
Mujer Z & -2.22 \\
Sig. asintót. (bilateral) & .02 \\
\hline Irresponsabilidad & \\
\hline N total Z & -.71 \\
Sig. asintót. (bilateral) & .47 \\
Varón Z & -.27 \\
Sig. asintót. (bilateral) & .78 \\
Mujer Z & -.37 \\
Sig. asintót. (bilateral) & .70 \\
\hline
\end{tabular}

Las medias de los ítems de la REP oscilan entre los valore 4.39 y $4.79(M=4.56, D T=0.78)$ indicando la existencia de una respuesta altamente positivahacia la relación intercultural. La REP, en su totalidad, aumentó más de 3 puntos (Pre $M=28,67$; Post $M=$ a 31.97).El comportamiento intercultural experimentó una subida con puntuaciones muy similares en chicos y chicas. Se observa una mejora en la dimensión ayuda, siendo dicho incremento mayor en varones que en mujeres. Por su parte, la irresponsabilidad (comportamientos menos deseados en el alumnado) ha subido ligeramente tanto en chicos como en chicas, si bien los valores resultantes se sitúan en valores de neutralidad respecto a dicha dimensión.

La Tabla 3 muestra las puntuaciones $Z$ y la Significación Asintótica de cada una de las comparaciones pretest-postest de la REP mediante rangos.

Tabla 3. Puntuaciones $\mathrm{Z}$ y significación asintótica bilateral (Sig. A. Bil.) de la prueba de Rangos de Wilcoxon de las variables a estudio.

\begin{tabular}{lc}
\hline & Postest - Pretest \\
\hline REP & \\
\hline N total Z & -3.60 \\
Sig. asintót. (bilateral) & .00 \\
Varón Z & -2.38 \\
Sig. asintót. (bilateral) & .01 \\
Mujer Z & -2.56 \\
Sig. asintót. (bilateral) & .01 \\
\hline Comportamiento Intercultural & \\
\hline N total Z & -3.52 \\
Sig. asintót. (bilateral) & .00 \\
Varón Z & -2.70 \\
Sig. asintót. (bilateral) & .00 \\
\hline
\end{tabular}

Se encontraron diferencias significativas entre el pretest y postest en la subescala REP de la sensibilidad intercultural, la escala Comportamiento intercultural y la subescala ayuda tanto en la muestra total como en varones y mujeres por separado.

Finalmente, en relación a las comparaciones en función del género, la Tabla 4 recoge las puntuaciones $\mathrm{Z}$ y significación asintótica bilateral (Sig. A. Bil.) de la prueba de $U$ de Mann-Whitney de cada una de las variables a estudio y en cada una de las administraciones de los cuestionarios.

Tabla 4. Puntuaciones $\mathrm{Z}$ y significación asintótica bilateral (Sig. A. Bil.) de la prueba de $U$ de Mann-Whitney de la REP (Sensibilidad Intercultural).

\begin{tabular}{lcc}
\hline & Pretest & Postest \\
\hline REP & & \\
\hline$U$ de Mann-Whitney & 46.500 & 46.500 \\
$Z$ & -2.137 & -2.168 \\
Sig. asintót. (bilateral) & .033 & .030 \\
\hline Comportamiento Intercultural & & \\
\hline$U$ de Mann-Whitney & 63.500 & 80.000 \\
$Z$ & -.791 & -.207 \\
Sig. asintót. (bilateral) & .429 & .836 \\
\hline Ayuda & & \\
\hline U de Mann-Whitney & 65.500 & 74.500 \\
$Z$ & -1.204 & -.768 \\
Sig. asintót. (bilateral) & .229 & .443 \\
\hline
\end{tabular}




\begin{tabular}{lcc}
\hline & Pretest & Postest \\
\hline Irresponsabilidad & & \\
\hline U de Mann-Whitney & 61.500 & 75.500 \\
$\mathrm{Z}$ & -1.316 & -.827 \\
Sig. asintót. (bilateral) & .188 & .408 \\
\hline
\end{tabular}

Los resultados de los análisis mostraron una valoración significativamente más elevada de las mujeres en pretest y postest en las REP de la sensibilidad intercultural, tanto en el pretest como en postest.

\section{Resultados cualitativos}

A continuación, se presenta la información cualitativa considerando las tres categorías extraídas del análisis de los diarios y entrevistas al profesorado, así como de las entrevistas al alumnado.

\section{Elementos metodológicos y su influencia en la interculturalidad}

A lo largo de la unidad, los docentes, como responsables del proceso de enseńanza-aprendizaje, reportaron una serie de decisiones metodológicas que causaron efectos, tanto positivos como negativos, a nivel intercultural. Dichas conexiones englobaron rasgos muy diversos. Es el caso de la secuencia didáctica, sobre la que el profesor inexperto aseguró que todas las fases del modelo fueron positivas a nivel intercultural, al tiempo que el experto manifestó que no hubo ni positivas ni negativas per se, sino que dependieron de su correcto funcionamiento, es decir, del cumplimiento de las características propias del modelo (roles, afiliación, registro de datos, etc.). Lo ejemplificó con la fase de práctica autónoma, donde un aspecto no controlado hubiese supuesto dejar autonomía absoluta al alumnado. Los profesores coincidieron en señalar a las fases introductoria y dirigida como determinantes, explicando que la inicial presentó mayores dificultades, siendo más crítica que las demás. Esto se debió, según la percepción de los docentes, a la gran variedad de aspectos por considerar (formación de equipos, aprendizaje de roles, dinámica de trabajo, etc.). Antes de aplicar la intervención, los profesores temían que los estudiantes, en el momento de la evaluación final, llevasen a cabo valoraciones injustas, con el propósito de perjudicar a los demás grupos, actuando en beneficio propio. Sin embargo, a pesar de estos temores iniciales, la fase en cuestión pasó inadvertida, sin incidencias de consideración. En este sentido, los docentes percibieron una alta competencia, tanto técnica como ética, por parte de los estudiantes al desempeñar los roles de duty team, tal y como afirmó el profesor experto: "Los jueces realizan su labor excepcionalmente". Su colega, además, destacó la normalidad y el entusiasmo duran- te el evento final. El propio alumnado así lo constató al decantarse, por unanimidad, por el evento final como momento favorito de la unidad. Al mismo tiempo, manifestó su buen hacer en las labores asumidas y el respeto hacia el resto de participantes. Estas labores aluden a los roles desempeñados por el alumnado, constituyendo otro elemento metodológico de gran relevancia a nivel intercultural. Desde la perspectiva docente, los roles resultaron muy provechosos para la mejora de la responsabilidad, así como para la organización del grupo-clase. Asimismo, los estudiantes también los evaluaron como imprescindibles. Pese a haber tenido experiencias previas con el modelo de ED, en un principio los estudiantes encontraron los roles algo complicados al desconocer la mayoría. Sin embargo, conforme avanzó la unidad y dominaron sus funciones, les resultó más sencillo aprenderlos y utilizarlos ya que tenían similitudes con otros empleados en contenidos deportivos. Entre ellos, el rol de director fue percibido, casi por unanimidad, como el más importante, debido en gran medida a las exigencias comunicativas y de responsabilidad que conlleva, argumentando varias razones, entre otras: " $E s$ el que informa a los compañeros" (Grupo 3) o "Es el que pone orden" (Grupo 5). De las palabras del profesorado se extrae que, a nivel intercultural, el alumnado de origen español logró desenvolverse mejor con los roles más directivos. Los docentes consideraron que los roles que implicaban mayor autoridad eran asumidos con mayor dificultad por parte del alumnado inmigrante. Entre las razones, destacaron las barreras idiomáticas y aspectos relacionados con la personalidad o el propio carácter del alumnado en cuestión. Sin embargo, a medida que avanzaba la unidad, en general los estudiantes manifestaron valerse de forma más eficaz con los roles, destacando su importancia para lograr una buena representación final y trabajar de manera organizada: "[Sin roles] sería un lío (...) Habría más discusiones” (Grupo 2). En esta misma línea, el alumnado destacó la importancia de grupos fijos para no descoordinarse a lo largo de la unidad. Al comienzo, uno de los docentes percibió que los grupos no habían sido del agrado de todos los estudiantes. Una vez avanzada la intervención, el profesor experto observó la incidencia de la organización social, justificando la disposición de grupos heterogéneos y destacando que influyeron positivamente en las relaciones interculturales: "El hecho de que [los grupos] estén equilibrados en género y nacionalidad implica algo positivo". Aseguró que de haber sido grupos homogéneos en cuanto a nacionalidad (esto es, grupos íntegramente compuestos por espańoles o marroquíes) se hubiese fomentado mayor competitividad.

\section{Efecto de la comunicación sobre la interculturalidad y el liderazgo}

La experiencia realizada, a juzgar por las observaciones docentes, provocó mejoras en la competencia comunicativa del 
alumnado. Antes de iniciar la unidad, el profesor experto declaraba que sería un aspecto clave por considerar: "Hay que solventar que no sea un hándicap el hecho de que alguien, en algún momento determinado, no controle la comunicación". Dentro de sus recomendaciones iniciales, abogaba por asegurar y facilitar la comprensión del material utilizado (hojas de anotación, instrucciones, etc.). Conforme fue avanzando la unidad, ambos docentes comprobaron que no influía demasiado. Tal como señalaron los mismos, quizá pudo deberse al nivel madurativo en el que se encontraba el alumnado ( $5^{\circ}$ curso), que poseía ya un dominio suficiente del lenguaje. Sin embargo, el profesor experto reportó un hecho distintiv oal observar que los estudiantes de nacionalidad española asumían mayores cuotas de liderazgo, precisamente, por controlar mejor la comunicación. Más allá de esa diferencia, aseguraron que la experiencia influyó de forma muy positiva en la competencia comunicativa del alumnado, sobre todo, en aquel de origen inmigrante: "He visto un extra de los inmigrantes respecto a los españoles", concluía el profesor experto. En este sentido, los docentes comentaron que la expresión corporal es un contenido en el que la comunicación está presente constantemente y asumió gran importancia a lo largo de toda la unidad: "La comunicación era el vehiculo", afirmaba el mismo profesor, aludiendo a aquella comunicación que debieron establecer durante el proceso de elaboración de la obra, tomando decisiones y llegando a acuerdos. En esta misma línea, el mismo profesor argumentó que durante la representación de las obras tuvo una gran relevancia la comunicación no verbal, al tratarse del mimo: "El hecho de no poder hablar (durante la obra) les ha vuelto más competentes". Así, estas mejoras comunicativas reportadas por el profesorado y el alumnado se refirieron, asimismo, a las mejoras derivadas del contenido y relacionadas con aspectos técnicos (gestos) propios de la actividad desarrollada. Estas experiencias de aprendizaje repercutieron sobre las habilidades creativas del alumnado. Ambos docentes observaron que, a diferencia con los deportes colectivos, en esta unidad el liderazgo no lo asumían los más habilidosos motrizmente, sino aquellos más creativos. Así lo comentó el docente inexperto: "Niños que antes no tenían tanto peso en otras unidades más deportivas, lo están teniendo ahora. No tanto por la habilidad motriz, sino por la creatividad". El profesor experto compartió ese parecer: "Donde veo más diferencia es que está cogiendo más liderazgo quien más creatividad tiene, cosa que en deportes no pasa”. Por escrito, lo sintetizó con mayor profusión, abogando por delimitar puntos de conexión entre comunicación, creatividad e interculturalidad: "El liderazgo lo tienen los más creativos y aquellos que tienen más ideas, independientemente de su nivel motriz que, normalmente, han sido de nacionalidad española, tanto niños como niñas".

\section{Clima social del aula e interculturalidad}

Como se apuntaba, una vez formados los grupos, se observaron discrepancias entre el alumnado, argumentando preferencias por afinidad. Sin embargo, el profesor experto se mostró convencido de que las relaciones iban a prosperar. Distintos comentarios por parte del profesor inexperto, ya durante el transcurso de la unidad, apuntaron a mejoras en las relaciones sociales: "Las relaciones son muy buenas"; "Las relaciones por nacionalidad, de momento, están siendo muy buenas. Hacen las sesiones muy agradables". Así lo avaló un grupo de alumnos al ser preguntados sobre el hecho de trabajar en un contexto intercultural: "Nos conocemos mejor y hacemos amigos" (Grupo 2). En ese sentido, emergió el concepto aprendizaje del propio alumnado, quien justificó los encuentros interculturales en cuanto entrañaron oportunidades para aprender de los demás. Un alumno lo fundamentó así: "Es una buena experiencia [...] porque podemos trabajar todos juntos de culturas [distintas]. Ellos aprenden un montón de cosas" (Grupo 4). Otro grupo añadió: "Ellos nos enseñan y nosotros les enseñamos" (Grupo 5). El profesor experto, por su parte, argumentó la importancia del contenido en las relaciones: "Está haciendo que sean mucho más mayores [...], a la vez que fluidas y positivas", llevándole a aconsejar este tipo de contenidos en contextos similares. Pero no solo se evidenció la relevancia del contenido de manera aislada, sino de su conjunción con el modelo utilizado: "Está haciendo que sea muy elevado el número de interacciones sociales. [...]Es un cóctel muy importante". Los docentes lo reconocen como una unión que ha favorecido la integración del alumnado extranjero. El incremento en las interacciones se tradujo, igualmente, en mayores relaciones colaborativas. Los profesores manifestaron que la idea de ayudarse estuvo presente a lo largo de toda la unidad, razonando que la colaboración no aumentó de manera exclusiva y significativa en términos interculturales, sino que permeó a todo el alumnado. Así lo confirmó el profesor inexperto, al asegurar que las ayudas entre el alumnado fueron más allá de la interculturalidad, sin ser necesaria una intervención específica que atendiese a las características referidas: "El concepto ayuda está saliendo de forma muy notable; todos se dan feedback, se ayudan mucho" apuntaba. Varios grupos de estudiantes reflejaron ejemplos de relaciones colaborativas al sostener que prestaban ayuda a los compañeros que tenían más dificultades. Esta pertinente colaboración repercutió, naturalmente, sobre el producto final. El profesor experto anticipó con acierto que las ayudas surgirían debido al objetivo común inherente al modelo, subrayando la importancia de aquella de tipo comunicativo: "Es máxima en contextos con inmigrantes". Otros ejemplos de aprendizaje dialéctico se dieron al observar cómo los estudiantes, por unanimidad, acudían al diálogo para solucionar los problemas que se iban encontrando. Al preguntarles si habían vivido algún conflicto, un grupo contestó: "Sí, un poqui- 
llo, pero lo hemos resuelto hablando. El papel de [alumna], que no salia mucho, lo hemos resuelto sacándola un poco más" (Grupo 2). El profesor inexperto sostuvo que, a través de esta unidad, se estrecharon mucho más los lazos entre el alumnado: "Ha sido una relación de acercamiento", comentó. Este aspecto fue percibido de forma análoga por el profesor experto quien indicó que alumnos que antes no estaban muy involucrados en el aula, han disfrutado siendo uno más en el grupo, con abrazos y sonrisas. Estas mejoras en las relaciones también se reflejaron en un ambiente cordial de trabajo, caracterizado por el respeto, el disfrute y la diversión. Al respecto, el profesor experto escribía lo siguiente: "Impera un respeto increíble a la hora de tomar acuerdos y opinar sobre la obra". De hecho, ambos docentes coincidieron al destacar las actitudes deferentes que mantuvieron los estudiantes respecto a la participación ajena: "Hay un máximo respeto por cada equipo que ha representado su obra" (profesor experto); "Las representaciones transcurren (...) con mucho respeto por parte de los observantes" (profesor inexperto).

\section{Discusión}

El objetivo de este estudio ha sido analizar en qué medida un plan de intervención sobre mimo basado en los principios del MED puede mejorar la competencia intercultural del alumnado en términos de sensibilidad y comportamiento interculturales. Las respuestas emocionales positivas a la sensibilidad intercultural del alumnado aumentaron de forma significativa en ambos géneros. Del mismo modo lo hicieron los comportamientos interculturales, en especial, las conductas de ayuda, sin que llevaran asociados cambios significativos en el factor de irresponsabilidad. Los estudiantes (autóctonos e inmigrantes), demostraron de manera bidireccional una gran disposición y disfrute a la hora de trabajar con compañeros de otras culturas, coincidiendo con la experiencia transnacional realizada por Hastie et al. (2013), donde los estudiantes valoraron de forma muy positiva sus encuentros virtuales con homólogos de otros países. Dichas mejoras se deberían poder objetivar a partir de dos aspectos, fundamentalmente. Por un lado, la idea de afiliación, determinante a la hora de crear sentido de pertenencia a nivel intergrupal y mejorar las relaciones entre sus componentes (Grant, 1992; MacPhail, Gorely, Kirky Kinchin, 2008). Por otro lado, la importancia del contenido trabajado, defendido por los docentes como un aspecto clave para generar entusiasmo en el alumnado (Calderón et al., 2013), así como interacciones constantes y positivas. Los resultados refuerzan la idea de que el MED incide positivamente en las relaciones entre iguales, tal como lo constataron estudios anteriores (Brock, Rovegno y Oliver, 2009; Cruz, 2008; García-López et al., 2012; Grant, 1992; Hastie, 1998; López et al., 2016; MacPhail, Kirk y Kinchin, 2004; O’Donovan, 2003). El presente estudio confirma di- chas mejoras y las extrapola por primera vez a los contextos multiculturales.

En segundo lugar, las relaciones de colaboración, manifiestas a lo largo de la intervención, han aumentado de forma general en todo el alumnado. Dicha habilidad se ha constatado a medida que avanzaba la unidad. Esto ha sido, en gran medida -y como hallaron Ka y Cruz (2006)-, debido al dominio eficaz de la responsabilidad asumida. Del mismo modo, como dedujeron Hastie et al. (2013), la idea de colaboración vino reforzada por aquella de afiliación que, en su caso, logró crear un sentido de pertenencia entre miembros de diferentes países, más allá de las fronteras y las distancias. Sin embargo, se encontraron diferencias en función del género en las REP en favor de las mujeres, tanto en el pretest como en el postest. Esta tendencia más positiva de las niñas a empatizar con otras realidades culturales fue subrayada en el estudio de Sanhueza (2010) e interpelan al desarrollo de intervenciones e innovaciones futuras docentes que puedan paliar estas desigualdades.

Uno de los aspectos más valorados a lo largo del estudio ha sido la comunicación, presentándose como uno de los elementos objeto de análisis. Resulta interesante observar cómo ha gravitado sobre el resto de elementos metodológicos. Los docentes reportaron sus efectos, tanto positivos como negativos, concluyendo que cuando no es bien planteada puede suponer dificultades añadidas al alumnado. Así pues, se antoja como necesaria una intervención que permita crear cauces comunicativos eficaces a partir de procesos participativos que encuentren en las decisiones consensuadas y en la colaboración sus rasgos más sobresalientes. Aquellos problemas que se puedan derivar de la interacción (rivalidad intra e intergrupal, amenaza del autoconcepto, autonomía, etc.) deben ser resueltos con aquellos recursos que nos ofrecen el propio MED y sus sucedáneos más próximos. Trascendiendo elementos metodológicos, se retiene a la expresión corporal como una herramienta eficaz, en sí misma, para el desarrollo de habilidades comunicativas. Los resultados del presente estudio van en la línea de estudios previos que ponen en valor el uso del MED para la implementación de la expresión corporal (Méndez-Giménez et al., 2017a; 2017b).

Por su parte, los resultados obtenidos en la dimensión irresponsabilidad (referidos a aquellos comportamientos más negativos del alumnado) hacen prever que la intervención no ha incidido en dicho aspecto. No obstante, los bajos niveles de fiabilidad de esta escala (entre .64 y .69) nos llevan a interpretar estos resultados con cautela. Los análisis cualitativos permiten afirmar que a lo largo de la intervención, no se observaron comportamientos negativos o irresponsables objeto de consideración; más bien ocurrió lo contrario. En todo caso, las discusiones que pudieron presentarse fueron resueltas mediante el diálogo, siendo un proceder reconocido por la amplia mayoría de los respondientes. 
Finalmente, resulta oportuno poner en tela de juicio algunos aspectos del estudio. No debemos obviar las características iniciales del contexto. Esto es, hemos partido de resultados muy positivos en materia de interculturalidad, tanto en sensibilidad como en comportamiento, evidenciando que el alumnado, en su mayoría, partía de un alto grado de familiarización con el hecho intercultural. Para superar dichas limitaciones, en futuras investigaciones, resultará provechos o considerar otros datos demográficos del alumnado, entre otros, (a) el tiempo de estancia en el país de acogida o (b) la experiencia previa con alumnado culturalmente diverso. Una segunda limitación estriba en que el estudio no fue diseñado, a priori, para obtener resultados a nivel intercultural. Debemos recordar que se trata de un estudio aprovechado coyunturalmente en el que se presentó la posibilidad de incluir y analizar la dimensión intercultural del aprendizaje. En este sentido, el presente estudio ha servido como pilotaje para futuras intervenciones. Una tercera limitación se da en el propio diseño cuasi-experimental del estudio, principalmente, por dos aspectos: (a) el número de participantes y (b) la carencia de grupo control. Futuros trabajos deberían ir orientados a superar dichas condiciones, así como a explorar diferentes contextos (alumnado de mayor edad -enseñanza secundariao centros con tasas de alumnado inmigrante más bajas).

\section{Aplicaciones prácticas}

Las necesidades educativas emergentes en materia de interculturalidad, y en vistas a un diseño mejorado, estriban en una serie de decisiones metodológicas por considerar. Dentro de las tareas organizativas iniciales, se ha constatado la importancia de apostar por grupos heterogéneos (Martínez de Ojeda, 2012; Siedentop, Hastie y van der Mars, 2011) que atiendan a numerosas variables, entre ellas, la diversidad cultural. Igualmente, la asignación de roles (que, en este caso, han constituido una doble responsabilidad), así como la afiliación, se confirman como dos elementos indispensables para la mejora de las relaciones sociales, colaborativas y comunicativas (Derri et al., 2014; MacPhail, Kirk y Kinchin, 2004; O’Donovan, 2003), así como para potenciar los niveles de sensibilidad y comportamiento interculturales en sus dimensiones positivas. Cabe recordar la gran apertura y disposición que demostró el alumnado en sus encuentros interculturales. Por su parte, el hecho de perseguir un objetivo común favorece la interdependencia positiva, lo que nos lleva a apostar por una intervención cuya fase de síntesis incluya un evento o representación final.

Respecto al contenido desarrollado, hemos visto que, más allá de la inusitada producción de investigaciones a partir de los postulados del MED que se sirven de contenidos deportivos como tema central (Brock, Rovegno y Oliver, 2009; Browne, Carlson y Hastie, 2004; Carlson, 1995; Cruz, 2008; Hastie, Sinelnikovy Guarino, 2009; Mowling, Brock y Hastie, 2006), la expresión corporal supone un recurso muy positivo para ser desarrollado a través del modelo referido (Calderón et al., 2013; Méndez-Giménez et al., 2017a; 2017b). Entre sus ventajas, destaca la mejora de la cohesión interna, la disminución de la competitividad y, no menos importante, la oportunidad que brinda a los practicantes menos hábiles motrizmente, pero más competentes a nivel creativo, de poder asumir mayores cuotas de protagonismo en sus aprendizajes, así como un mayor liderazgo a nivel intergrupal (Hastie, 1998). Los docentes observaron que la habilidad motriz perdía relevancia en pos de la creatividad, lo que nos lleva a asumir que este tipo de intervenciones promueve el desarrollo del pensamiento divergente (Méndez-Giménez, 2011). Considerando estas observaciones, futuras líneas de investigación deberían ir dirigidas a contextos en los que no se presenten barreras comunicativas pudiendo aislar este aspecto y, de este modo, sondear las correlaciones directas entre creatividad e interculturalidad.

\section{Conclusiones}

El Modelo de Educación Deportiva parece provocar un efecto positivo en algunas dimensiones de la sensibilidad y competencia intercultural del alumnado de primaria al abordar el contenido del mimo. La característica de afiliación podría ser clave en la mejora de las relaciones entre estudiantes autóctonos e inmigrantes. La expresión corporal se ofrece como una herramienta eficaz para el desarrollo de habilidades comunicativas, carentes en el alumnado inmigrante.

\section{Referencias}

1. Arslan, Y., Günçavdi, G., y Polat, S. (2014). The impact of a peace education program at university on university student's intercultural sensitivity. Procedia - Social and Behavioral Sciences, 174,2301-2307.

2. Banks, J. A. (2002). An introduction to multicultural education (3a ed.). Boston: Allyn and Bacon.

3. Barrett, T. (2005). Effects of cooperative learning on the performance of sixth-grade physical education pupils. Journal of Teaching in Physical Education, 24, 88-102.

4. Barrett, T. M. (2000). Effects of two cooperative learning strategies on academic learning time, student performance and social behavior of sixth-grade Physical Education students. [Tesis doctoral]. Universidad de Nebraska. En Dyson, B. (2002). The implementation of cooperative learning in an elementary school Physical Education program. Journal of Teaching in Physical Education, 22(1), 69-85.

5. Bisquerra, R. (Coord.) (2004). Metodología de la investigación educativa. Madrid: La Muralla.

6. Brock, S. J., Rovegno, I., y Oliver, K. L. (2009). The influence of student status on student interactions and experiences during a sport education unit. Physical Education y Sport Pedagogy, 14(4), 355-375.

7. Browne, T. B., Carlson, T. B., y Hastie, P. A. (2004). A comparison 
of rugby seasons presented in traditional and sport education formats. European Physical Education Review, 10(2), 199-214.

8. Burden, J.W., Hodge, S.R., O'Bryant, C.P., y Harrison, L. (2004). From Colorblindness to Intercultural Sensitivity: Infusing Diversity Trainingin PETE Programs. Quest, 56(2), 173-189.

9. Calderón, A., Hastie, P., Liarte, J. P., y Martínez de Ojeda, D. (2013). El modelo de educación deportiva y la enseñanza de la danza. Una experiencia de bachillerato. Tándem. Didáctica de la Educación Física, 93-98.

10. Carlson, T. B. (1995). Now I think I can: The reaction of eight lowskilled students to sport education. ACHPER Health Lifestyles Journal, 42(4), 6-8.

11. Chen, G. M., y Starosta, W. J. (1996). Intercultural communication competence: A synthesis. En B. Burleson (Ed.), Communication Yearbook 19, 353-383. Thousand Oaks: Sage.

12. Chen, G. M., y Starosta, W. J. (1997). A review of the concept of intercultural sensitivity. Human Communication, 1, 1-16.

13. Chen, G.M., y Starosta, W. J. (2000). The development and validation of the Intercultural Sensitivity Scale. Human Communication, 3(1), $2-14$.

14. Chongruska, D., Prinyapola, P., Wadengb, Y., y Padungponga, C. (2010). Storytelling: program for multicultural understanding and respect among Thai-Buddhist and Thai-Muslim students. Procedia - Social and Behavioral Sciences, 5, 282-288.

15. Cohen, L., y Manion, L. (2002). Métodos de investigación educativa. Madrid: La Muralla.

16. Cruz, A. (2008). The experience of implementing sport education model. Journal of Physical Education y Recreation (HK), 14(1), 18-31.

17. Derri, V., Kellis, I., Vernadakis, N., Albanidis, E., y Kioumourtzoglou, E. (2014). The Effect of an Intercultural Physical Education Program in Comparison to the Typical One on Students' Social Skills Learning. Journal Human Sport Exercise 9(1), 91-102.

18. Dyson, B. (2005). Integrating Cooperative Learning and Tactical Games Models: Focusing on Social Interactions and Decision Making. En Teaching Games for Understanding. (pp. 149-168). Theory, research and practice. Champion, Il.: Human kinetics

19. Dyson, B. (2002). The Implementation of Cooperative Learning in an Elementary Physical Education Program. Journal of Teaching in Physical Education, 22, 69-85.

20. Ennis, C. D., y Chen, S. (2012). Interviews and focus groups. En Armour, K., Macdonald, D. (Ed.), Research Methods in Physical Education and Youth Sport. (p. 217-236). New York: Routledge.

21. Erickson, F. (1989). Métodos Cualitativos de Investigación Sobre la Enseñanza. En Wittrock, Merlin C. (Ed.). La Investigación de la Enseñanza, II - Métodos Cualitativos y de Observación. Barcelona: Ediciones Paidós.

22. Fernández-Río, J. (2003). El aprendizaje cooperativo en el aula de educación física para la integración en el medio social: análisis comparativo con otros sistemas de enseńanza y aprendizaje. [Cdrom]. Valladolid: La Peonza.

23. García-López, L.M., Gutiérrez, D., González-Víllora, S., y Valero, A. (2012). Cambios en la empatía, la asertividad y las relaciones sociales por la aplicación del modelo de instrucción educación deportiva. Revista de Psicología del Deporte, 21(2), 321-330.

24. Gordon, B. (2009). Merging teaching personal and social responsibility with sport education: A marriage made in heaven or hell? ACHPER Healthy Lifestyles Journal, 56(3/4), 13-16.

25. Grant, B.C. (1992). Integrating sport into the physical education curriculum in New Zealand secondary schools. Quest, 44, 304-316.

26. Hambleton, R., Merenda, P., y Spielberger, C. (Eds.). (2005). Adapting educational and psychological tests for cross-cultural assessment. Mahwah, NJ: Erlbaum.

27. Hastie, P. (1998). Applied benefits of the sport education model. JOPERD: The Journal of Physical Education, Recreation y Dance, 69(4), 24.

28. Hastie, P., Farias, C., y Gutiérrez, D. (2013). Students' and teachers' re- sponses to a transatlantic sport education league. Journal of the International Society for Comparative Physical Education and Sport, 35(2), 22-33.

29. Hastie, P., Sinelnikov, O. A., y Guarino, A. J. (2009). The development of skill and tactical competencies during a season of badminton. European Journal of Sport Science, 9(3), 133-140.

30. Hellison, D. (2011). Teaching Personal and Social Responsibility through physical activity (3a ed.). Champaign, IL: Human Kinetics.

31. Hernández, J. A. (2011). La competencia intercultural en el alumnado de educación primaria. (Tesis doctoral). Universidad de Alicante.

32. Ka, L. C., y Cruz, A. (2006). The effect of sport education on secondary six students' learning interest and collaboration in football lessons. Journal of Physical Education y Recreation (HK), 12(2), 13-22.

33. Kellis I., Vernadakis N., Albanidis E., Derri V., y Kourtesses T. (2010). The development of a student's behaviors' self-evaluation scale (SBSS) in multicultural physical education class settings. Educational Research and Review, 5(11), 637-645.

34. Kouli, O. y Papaioannou, A.G. (2009). Ethnic/cultural identity salience, achievement goals and motivational climate in multicultural physical education classes. Psychology of Sport and Exercise, 10, 45-51.

35. López Sánchez, G. F., López Sánchez, L. \& Díaz Suárez, A. (2016). Effects of a physical activity program on the general dynamic and segmentary coordination of children with ADHD. Journal of Sport and Health Research, 8(2), 115-128.

36. MacPhail, A., Kirk, D., y Kinchin, G. D. (2004). Sport Education: promoting team affiliation through Physical Education. Journal of Teaching in Physical Education, 23(2), 106.

37. MacPhail, A., Gorely, T., Kirk, D., y Kinchin, G. (2008). Children's experiences of fun and enjoyment during a season of sport education. Research Quarterly for Exercise and Sport, 79, 344-355.

38. Malik (2002). Competencias interculturales. Proyecto docente. Madrid: UNED.

39. Mandigo, J., y Corlett, J. (2010). Teaching games for understanding of what? TGfU's role in the development of physical literacy. En J. I. Butler y L. L. Griffin (Eds.), More teaching games for understanding. Moving globally. (pp. 69-78). Champaign, IL: Human Kinetics.

40. Martínez de Ojeda, D. (2013). El modelo de enseñanza de Educación Deportiva (Sport Education): Aprendizaje, enseñanza y aplicaciones prácticas (Tesis doctoral). Universidad Católica San Antonio - Murcia, España.

41. Martínez de Ojeda, D., Méndez-Giménez, A. y Valverde, J. (2016) Efectos del modelo Educación Deportiva en el clima social aula, la competencia percibida y la intención de ser físicamente activo: un estudio prolongado en primaria. Sport TK. Revista Euroamericana de Ciencias del Deporte, 5(2), 153-166.

42. Massot, I.; Dorio, I. y Sabariego, M. (2004). Estrategias de recogida y análisis de la información. En Bisquerra, R. (Coord.). Metodología de investigación educativa. Barcelona: La Muralla, 204-219.

43. Méndez-Giménez, A. (2011). El proceso de la creación de juegos de golpeo y fildeo mediante la hibridación de modelos de enseñanza. Ágora para la educación Física y el Deporte, 13(1), 55- 85.

44. Méndez-Giménez, A. y Martínez de Ojeda, D., y Valverde, J.J (2017a). Efecto de una temporada de Educación Deportiva dedicada al mimo en la autorregulación (evaluación), la diversión y la competencia del alumnado. EmásF, 44, 21-40.

45. Méndez-Giménez, A. y Martínez de Ojeda, D., y Valverde, J.J (2017b), Inteligencia emocional y mediadores motivacionales en una temporada de Educación Deportiva sobre mimo. Ágora para la Educación Física y el Deporte, 19(1), 52-72.

46. Mowling, C. M., Brock, S. J., y Hastie, P. (2006). Fourth grade students'drawing interpretations of a sport education soccer unit. Journal of Teachingin Physical Education, 25(1), 9-35.

47. Muniategi, E., Sianesb, A. y López, I. (2014). Facing FGM/C through Intercultural Education: A Methodology for Secondary School Communities. Procedia - Social and Behavioral Sciences, 132, 557-563. 
48. O’Donovan, T. M. (2003). A changing culture? Interrogating the dynamics of peer affiliations over the course of a sport education season. European Physical Education Review, 9(3), 237-251.

49. Padró, C. (2004). Aportaciones de la comunicación intercultural en el ámbito de la salud. Educare21, 10.

50. Pérez, S. (2013). Evaluación de un programa para el desarrollo de la competencia intercultural a través de la educación rítmica en educación primaria. Revista Electrónica de Investigación Educativa, 15(3), 124-138.

51. Porlán, R.,y Martín, J. (1991). El diario del profesor: un recurso para la investigación en el aula. Sevilla: Ed. Díada.

52. Richardson, M., y Oslin, J. (2003). Creating an authentic dance class using sport education. Journal of Physical Education, Recreation, and Dance, 74(7), 49-55.

53. Rodrigo, M. (1996). Los estudios de comunicación intercultural. Revista de Estudios de Comunicación, 1-6.

54. Rodríguez, H. (2015). Capítulo 3. Marco de referencia internacional y modelos emergentes en educación inclusiva. Análisis sobre el enfoque inclusivo en educación. En Escarbajal, A. (Ed.). Comunidades interculturales y democráticas. Madrid: Narcea.

55. Sanhueza, S.V. (2010). Sensibilidad intercultural: un estudio exploratorio con alumnado de educación primaria y secundaria en la provincial de Alicante. Tesis doctoral. Universidad de Alicante.

56. Sanhueza, S.V. y Cardona, M.C. (2009). Evaluación de la sensibilidad intercultural en alumnado de educación primaria escolarizado en aulas culturalmente diversas. Revista de Investigación Educativa, 27(1), $247-$ 262.

57. Santos, M.A. y Lorenzo, M. (2015). Capítulo 5. El éxito educativo desde la pedagogía intercultural. Formación con valor añadido para un mundo global. En Escarbajal, A. (Ed.). Comunidades interculturales y democráticas. Madrid: Narcea.
58. Sheppard, J. (2014). Personal and Social Responsibility through Game Play: Utilizing the Teaching Games for Understanding Instructional Model (Tesis doctoral). Universidad de Toronto.

59. Siedentop, D., Hastie, P. A., y H. van der Mars (2011). Complete Guide to Sport Education, Second Edition. Champaign, IL: Human Kinetics.

60. Thorpe, R.D. (1992). The psychological factors underpinning the "teaching for understanding games" movement. En Williams, T., Almond, L. y Sparkes, A. (Eds.), Sport and physical activity: Moving toward excellence (AIESEP World Convention, Loughbough, Reino Unido). (pp. 209-218). Londres: E y F.N. Spon,

61. Tsangaridou, N., y Lefteratos, C. (2013). Elementary Students' Views and Experiences on Sport Education in Cyprus. Advances in Physical Education, 3(1), 28-35.

62. UNESCO (2005). Guidelines for Inclusion. Ensuring Access to Education for All. Orientaciones para la inclusión. Asegurar el acceso a la Educación para Todos. Paris, UNESCO.

63. Velázquez, C. (Coord.) (2010). Aprendizaje cooperativo en Educación Física. Fundamentos y aplicaciones prácticas. Barcelona: INDE.

64. Vilà, R. (2003). La comunicación intercultural en Educación secundaria Obligatoria: Test de competencia comunicativa intercultural. En L. P. Buendía, T; González, D. y Sánchez, C.A. (Ed.) Investigación y Sociedad(pp. 1181-1188). Granada: Aidipe.

65. Vilà, R. (2005). La competencia comunicativa intercultural. Un estudio en el Primer Ciclo de la ESO. Tesis doctoral. Barcelona: Universidad de Barcelona.

66. Wallhead, T. L., Hagger, M., y Smith, D. T. (2010). Sport education and extracurricular sport participation: An examination using the trans-contextual model of motivation. Research Quarterly in Exercise and Sport, 81, 442-455. 